\title{
La tercera antinomia, la razón y la libertad. Revisión de la solución kantiana a la antinomia de la libertad
}

\author{
Miguel Alejandro Herszenbaun \\ UBA / CONICET/ CIF / GEK (Argentina) \\ herszen@hotmail.com
}

Recibido: 08/07/2015

Aceptado: $15 / 08 / 2015$

\section{Resumen}

El presente trabajo analiza la solución kantiana a la tercera antinomia o antinomia de la libertad. Nos proponemos defender la coherencia entre el problema planteado por Kant y la solución ofrecida. Para ello, sostendremos la necesidad de reformular el problema cosmológico planteado en términos realista-trascendentales a los términos propios del idealismo trascendental. A través de esta reformulación lograda gracias a la introducción de la libertad práctica, el problema continuará siendo de carácter estrictamente cosmológico. Sin embargo, se hará necesario explicar por qué Kant introduce consideraciones sobre la razón práctica en un contexto que, según nuestra lectura, es cosmológico. Sostendremos que Kant introduce estas referencias por motivos únicamente argumentales que no obligarían a Kant a realizar mayores consideraciones sobre la razón práctica en el contexto de la tercera antinomia.

Palabras clave: Libertad - Antinomia - Idealismo Trascendental - Razón - Cosmología.

\begin{abstract}
This paper studies the kantian solution to the third antinomy or freedom's antinomy. I intend to defend the inner coherence between the problem presented by Kant and its solution. Therefore, I claim the need to re-enunciate the cosmological problem, enunciated in terms of transcendental realism, into terms of transcendental idealism. By this re-enunciation made by the notion of practical freedom, the problem remains
\end{abstract}


strictly cosmological. Nevertheless, it will be necessary explain why Kant introduces references about the practical reason in a context that should be cosmological. I claim that Kant introduces these references for strictly argumental reasons that do not obly Kant to undergo any further considerations about the practical reason.

Keywords: Freedom - Antinomy - Transcendental Idealism - Reason - Cosmology.

\section{CV}

Miguel Alejandro Herszenbaun es Profesor de Filosofía (UBA), Abogado (UBA) y Becario Doctoral del CONICET. Es docente del Departamento de Derecho Penal de la Facultad de Derecho de la Universidad de Buenos Aires. Es miembro de numerosos grupos de investigación, miembro del Centro de Investigaciones Filosóficas (CIF) y del Grupo de Estudios Kantianos (GEK UBA). Ha participado de varios congresos y eventos académicos, ha publicado artículos en prestigiosas revistas internacionales y capítulos de libro. Se dedica especialmente a la filosofía moderna y al idealismo alemán.

\section{Introducción}

La tercera antinomia ha sido reiteradamente interpretada desde una perspectiva práctica. Autores como J. Bennett ${ }^{1}$ y P. Strawson ${ }^{2}$ han sostenido que este conflicto de

\footnotetext{
1 “Claramente, Kant está poniendo toda su confianza en la doctrina de la Analítica de que toda experiencia posible debe adecuarse a la causalidad de la naturaleza. La balanza se inclinan en favor de la antítesis. Ella está apoyada por una de las doctrinas más fuertemente sostenidas de Kant, mientras que el argumento de la Tesis es inconexo y oscuro, y la falta de confianza de Kant en él se ve en el hecho de que él no vuelve a aludir a él otra vez luego de su enunciación inicial.

"Su verdadera preocupación en este área, sin embargo, es menos imparcial. Su apoyo a la antítesis tiene la base que aparenta tener; pero su simpatía por la Tesis, juzgando de acuerdo con el tratamiento siguiente de la tercera antinomia, tiene sus raices en una consideración no sobre la suficiencia causal o la completitud de las cadenas causales o algo de este estilo, sino sobre la libertad humana. Lo que compromete al interés de Kant en la Tesis no es tanto de que ella "ofrece un punto de descanso en el entendimiento escrutador en la cadena de causas" sino que ella ofrece "una liberación de la coerción".

"[...] Kant no está manipulando dos temas en uno en su tratamiento de la tercera antinomia, puesto que casi completamente ignora el argumento de la Tesis tal como está formulado, concentrándose en cambio en la libertad de "mí mismo pensante", i.e. en la consideración de que "el albedrío humano es libre" [...].

"Kant no deja simplemente de lado el tema con el que comenzó, tomando en cambio el problema de la libertad humana. En su "Observación" a la Tesis, Kant trata de vincular dos temas a fin de justificar su viraje de uno a otro. [...]

"[...] No hay justificación filosófica para dirigirse hacia la libertad humana a través del argumento de la Tesis que Kant nos ha dado". La traducción es nuestra. BENNETT, Jonathan, Kant's Dialectic, Cambridge, Cambridge University Press, 1974, 188-189.
} 
la razón teórica presenta, en su verdadero significado, un problema estrictamente práctico que debería ser efectivamente abordado en el marco de la filosofía práctica. Se ha llegado a afirmar incluso que el verdadero móvil que guía a Kant hacia la tercera antinomia es su intención de abordar el problema de la libertad humana y no un pretendido problema cosmológico. En relación con estas interpretaciones, suele cuestionarse la concordancia entre el problema planteado y la solución ofrecida por Kant, teniendo en cuenta que mientras la "Antinomia de la razón pura" presenta cuatro conflictos de naturaleza cosmológica, la solución del tercer conflicto abordaría una temática perteneciente a la filosofía práctica. En este mismo sentido, se ha entendido que las nociones de razón y libertad analizadas en la solución a la tercera antinomia son, en efecto, de carácter netamente práctico.

En lo siguiente, propondremos otra interpretación de la tercera antinomia. En primer lugar, intentaremos demostrar que el problema planteado y su solución son, en todo caso, cosmológicos. Esto exigirá mostrar que la solución aborda el mismo problema que se planteara en la antinomia, sólo que ahora reformulado en términos idealistas trascendentales. En segundo lugar, nos ocuparemos de las nociones de razón y libertad allí consideradas. A fin de mostrar que el problema abordado y resuelto es en todo momento de carácter cosmológico, demostraremos que su solución no requiere mayores consideraciones sobre el uso práctico de la razón pura ${ }^{3}$. Con esto

\footnotetext{
2 “Pero resulta suficientemente obvio cuál, en el caso de la libertad contra la causalidad universal, es el [factor] fundamental. No tiene nada que ver con los intereses de la razón teorética. Tiene que ver con los intereses de la razón pura práctica, i.e. de la moralidad. Kant está ansioso por mostrar que la determinación causal de todo evento por las condiciones temporalmente antecedentes no es compatible con la idea de que ciertos eventos tengan otro tipo de causa, la cual, como perteneciente a la esfera nouménica, se encuentra exenta de la condición del tiempo y puede ser pensada como "actuando" libremente. [...] y es algo que la moralidad parece demandar, a pesar de que no podemos invocar conocimiento teórico al respecto". Aquí la razón de la "nueva" solución es clara: es la solución a un nuevo conflicto -un conflicto no presente en los argumentos de la tercera antinomia en lo absoluto" STRAWSON, Peter, The bounds of sense, London and New York, Routledge, 1966, 213. La traducción es nuestra.

${ }^{3}$ En la Crítica de la razón práctica Kant afirma que "[e]n éste [uso práctico de la razón] ocúpase la razón con fundamentos de determinación de la voluntad [...]. Pues ahí puede al menos la razón bastar para la determinación de la voluntad [...]" (AA, 5, 15; KANT, Immanuel, Crítica de la razón práctica, trad. de E. Miñana y Villasagra y Manuel García Morente, México DF, Ed. Porrúa, 1972, 101). De igual modo, se sostiene que "[s]i se admite que la razón pura puede encerrar en sí un fundamento práctico, es decir, bastante para la determinación de la voluntad [...]" (AA, 5, 19; Op. Cit. KANT, Immanuel, Crítica de la razón práctica, 103). En este sentido, A. Nuzzo explica que "la razón es práctica en la medida en que el peculiar tipo de causalidad ejercitado por ella, esto es causalidad por libertad, es dirigido no hacia
} 
queremos decir que aunque Kant introduzca la noción de libertad práctica, la solución de la tercera antinomia no exige mayores consideraciones sobre la razón pura práctica. Kant pretende demostrar que sería posible admitir a la vez una causalidad por libertad junto con una causalidad por naturaleza. $Y$, para esto, le basta con ofrecer buenos argumentos para admitir el carácter determinante de la razón sobre la conducta humana (como una prueba a favor del carácter inteligible del hombre). En otras palabras, no es necesario demostrar que esta facultad es la razón pura práctica, basta con dar buenos motivos para que el lector admita que la razón podría determinar la conducta ${ }^{4}$. De esta forma, se demostraría que el problema planteado y su solución son congruentes y que no se trata aquí de una cuestión práctica, sino cosmológica.

\section{Sobre la reformulación idealista trascendental del problema cosmológico metafísico dogmático}

objetos sino hacia la voluntad. [...] La causalidad de la razón en tanto razón pura práctica consiste en la inmediata determinación de la voluntad" (NUZZO, Angelica, Kant and the unity of reason, Indiana, Purdue University Press, 2005, 48). La traducción es nuestra.

${ }^{4}$ En el mismo sentido, nos interesa la posición de Allison, quien señala que aquí no se trataría de la determinación de la razón por medio de principios morales, sino sólo de una razón capaz de adoptar, espontáneamente, principios en general. H. Allison ha defendido esta posición al afirmar que "la afirmación de que imponemos imperativos en 'todo lo práctico' y que esta imposición siempre es considerada como una expresión de la 'causalidad' de la razón, subraya el punto anteriormente enfatizado de que la concepción kantiana de la libertad práctica no es una concepción del mero actuar moral. 'Lo práctico' a que Kant se refiere incluye las decisiones morales, pero no se limita a ellas" (ALLISON, Henry, El idealismo trascendental de Kant: una interpretación y defensa, Barcelona, Anthropos, 1992, 488). También, según el mismo autor, la libertad de la razón se encuentra relacionada con la espontaneidad de la misma al determinarse por principios que exceden el ámbito de la sensibilidad (ALLISON, Henry, Kant's theory of freedom, Cambridge, Cambridge University Press, 1990, 37-39). Esta posición también ha sido sostenida por Chong-Fuk Lau, quien ha planteado que la libertad práctica aquí abordada debe ser entendida como espontaneidad de la razón o la determinación de la razón según principios inteligibles, y que viene a responder la pregunta por la fundamentación de la objetividad, esto es, explica el origen o fundamento no sensible del fenómeno (Lau, Chong Fuk, "Freedom, spontaneity and the noumenal perspective", Kant-Studien, 99, 2008, 335). No obstante, como se verá más adelante, discrepamos en que dicho fundamento inteligible lo sería del mundo fenoménico como un todo. La causa inteligible no es causa del mundo como un todo, pues ni hay un mundo como un todo, ni tal causa libre es causa de todo fenómeno en el mundo, sino de ciertos fenómenos concretos en particular.

En todo caso, consideramos que para comprender la solución de la tercera antinomia en términos estrictamente cosmológicos no es necesario decidir si la razón considerada en la solución es una facultad autodeterminada sólo en el marco de la moralidad o en un marco más amplio en el cual se incluirían la adopción de principios teoréticos. Alcanza con comprender la función argumental de introducir la razón en este contexto: se pretende demostrar que es admisible la aptitud causal de la razón sobre los fenómenos, aun cuando todavía Kant no explique por medio de qué principios ocurre esto. 
A pesar de la interpración práctica propuesta por los autores mencionados, sostenemos que puede defenderse una lectura estrictamente cosmológica de la tercera antinomia. Dicha lectura implicaría considerar que el problema presentado en la antinomia versa sobre el origen del mundo y que su solución es congruente con el problema planteado. Esta congruencia es probada a través de lo que hemos llamado una reformulación idealista trascendental del problema cosmológico. Tal reformulación consiste en abordar el problema enunciado en términos metafísicodogmáticos, y enunciarlos en términos idealistas trascendentales. Veamos someramente lo fundamental de esta reformulación ${ }^{5}$.

La reformulación a que hacemos referencia consiste fundamentalmente en reelaborar el problema de tal modo que pueda ser formulado en términos congruentes con el idealismo trascendental y éste pueda dar una respuesta positiva. En otras palabras, en tomar la pregunta planteada en el tercer conflicto cosmológico, formulada en términos realista-trascendentales, y enunciarla en términos idealistatrascendentales, subsanando así los sin sentidos que tendría la posición realista trascendental. Sólo ante esta reformulación el problema se resuelve y encuentra respuesta, pues gran parte de la solución consiste en demostrar que la pregunta misma se encontraba mal formulada. Dicha reformulación consiste en convertir la pregunta por la existencia o inexistencia de un origen o causa incondicionada, libre y primera del mundo en el curso del tiempo en la pregunta por la causa incondicionada de cada fenómeno en particular o, al menos, de ciertos fenómenos en particular. Expliquemos brevemente por qué y cómo se realiza dicha reformulación.

La "Dialéctica trascendental" tiene como tarea desarticular la ilusión trascendental en la que la razón cae por su propia naturaleza y sobre la cual se fundan las metafísicas especiales racionalistas. La idea de alma, sobre la cual se elabora la psicología racional, es abordada en los "Paralogismos de la razón pura". El "Ideal de la razón pura" se ocupa de abordar la idea de Dios, sobre la cual se construye la teología racional. La "Antinomia de la razón pura" se ocupa de la idea de mundo, ie. la idea de

\footnotetext{
${ }^{5}$ Más detalles en HERSZENBAUN, Miguel, "Razón especulativa y la libertad práctica en la Dialéctica Trascendental" en Pulley, R., Martinez Saez, N. (eds.) Lenguaje, voluntad e igualdad en la sociedad moderna: homenaje a Jean-Jaques Rousseau: Actas III Jornadas Nacionales de Filosofía Moderna, Mar del Plata, Universidad Nacional de Mar del Plata, 2012, pp. 279-287.
} 
la totalidad absoluta de las condiciones del fenómeno en general. La "Antinomia" desarticulará las ilegímitas pretensiones gnoseológicas de esta supuesta disciplina metafísica, presentando las inevitables contradicciones en las que la razón cae cuando pretende obtener conocimiento a priori de un supuesto objeto concordante con esta idea de mundo.

La antinomia consta de cuatro conflictos en los cuales se pregunta por la serie de condiciones del fenómeno según cada una de las categorías. Cada conflicto consta de dos posiciones, una que sostiene la finitud de la serie de condiciones -tesis- y otra que defiende su infinitud -antítesis-. Kant caracteriza a las dos primeras antinomias como matemáticas. Esto se debe a que el tipo de enlace involucrado en ellas es de carácter homogéneo ${ }^{6}$. En ellas, se trata de la cantidad y cualidad y lo sintetizado debe ser del mismo género. La solución que Kant encuentra para estas antinomias es rechazar ambas posiciones. Lo que en un principio se presenta como una contradicción, Kant lo revela como una oposición dialéctica ${ }^{7}$. Esto es, no una contradicción en la que al menos una de ambas afirmaciones debe ser cierta, sino como una oposición en la que ambas pueden ser falsas.

La clave de esta solución consiste en revelar el presupuesto realista trascendental que se escondía detrás de ambas afirmaciones. Ambas posiciones suponen sin más que el mundo es una cosa en sí. Así, afirman (primera antinomia) que es en sí mismo finito o infinito (en cuanto a su cantidad, es decir, en tiempo o espacio). O afirman (segunda antinomia) que el mundo consta en sí mismo de partes últimas o de infinitas partes. En los dos casos se presupone algo que las hace falsas a ambas que el mundo es en sí- ${ }^{8}$.

Pero, para adoptar esta perspectiva que permite resolver el enigma planteado, Kant debe poder ver el problema desde fuera del marco en el que el problema se plantea. En otras palabras, debe ver el problema planteado desde fuera del realismo trascendental. El realismo trascendental presupone que los fenómenos son cosas en

\footnotetext{
${ }^{6}$ A529/B557. Todas las citas de la Crítica de la razón pura de Kant se hacen indicando la primera y segunda edición (A/B) seguido del número de paginación. La traducción es de Mario Caimi, Kant, Immanuel, Crítica de la razón pura, Buenos Aires, Colihue, 2007.

${ }^{7}$ A504/B532

${ }^{8}$ A504-5/B532-3.
} 
si $^{9}$. Desde esta perspectiva, el mundo como serie completa de condiciones del fenómeno debe ser algo en sí mismo con independencia de la facultad representativa del sujeto. $Y$, en consecuencia, debe ser finito o infinito en el tiempo y en el espacio. Rechazando que el mundo sea algo en sí mismo más allá de nuestras aptitudes epistemológicas, se puede negar ambas posiciones.

En lo que se refiere a las antinomias dinámicas (tercero y cuarto conflicto), la solución es diferente ${ }^{10}$. Una primera respuesta debería seguir los mismos lineamientos de la solución de las antinomias matemáticas. De hecho, Kant hace una sugerencia en este sentido (A505/B533). Y, en efecto, en tanto los adversarios allí enfrentados presuponen que el mundo es algo en sí mismo, se puede dar por falsas ambas afirmaciones y resolver el enigma del mismo modo que se lo hiciera en los casos anteriores.

Sin embargo, la tercera y cuarta antinomia suponen ciertos temas especulativos y prácticos que Kant no quiere desechar. En el caso que nos interesa, la tercera antinomia, Kant no tiene la intención de desechar sin más la posibilidad de la libertad humana. Por lo que, abordando el carácter dinámico del enlace allí considerado encuentra una solución que permite aceptar la posibilidad de la compatibilidad entre causalidad natural (o una serie infinita de causas mecánicas) y causalidad por libertad (causa incausada).

Veamos la tercera antinomia. Ella presenta la posición de la tesis, según la cual "[l]a causalidad según leyes de la naturaleza no es la única de la cual puedan ser

\footnotetext{
${ }^{9}$ A491/B519.

${ }^{10}$ Según Röttges, mientras Kant resuelve las dos primeras antinomias con una respuesta bajo la forma caracterizada como "ni...ni..." -esto es, rechazando ambas proposiciones-, las dos antinomias restantes son resueltas bajo la forma "tanto...como también..." -admitiendo las pretensiones de ambas posiciones y logrando reconciliarlas-. Para lograr esto, Kant debe introducir la distinción entre categorías matemáticas y dinámicas. El autor sostiene que dicha distinción es introducida exclusivamente para poder resolver la "Tercera antinomia" salvando la posiblidad de la libertad humana. Afirma el autor que esta distinción no cumple ninguna otra función a lo largo de toda la Crítica. Cfr. RÖTTGES, H., "Kants Auflösung der Freiheitsantinomie", Kant-Studien, 65, 1974, 41-42. Por su parte, respecto de la resolución del tercer conflicto cosmológico Grier sostiene que "la resolución de esta antinomia consiste en conceder la legitimidad de ambas posiciones, mientras se niega la absoluta universalidad de cada una de las conclusiones. [...] [E]n la suposición del realismo trascendental, tanto la naturaleza como la libertad parecen ser derribadas. Para evitar esta conclusión, Kant apela nuevamente al idealismo trascendental, el cual es supuesto para rescatar a la razón de este conflicto" GRIER, Michelle, Kant's doctrine of transcendental illusion, Cambrdige, Cambridge University Press, 2001, 217218. La traducción es nuestra.
} 
derivados todos los fenómenos del mundo. Es necesario, para explicarlos, admitir además una causalidad por libertad"11. Esto implica suponer una causa incondicionada y libre en la serie que constituye al mundo, causa de la cual se seguiría la totalidad de los acontecimientos del mundo. A esta tesis se opone la antítesis que afirma que "[n]o hay libertad, sino que todo en el mundo acontece solamente según leyes de la naturaleza"12. Así, la totalidad de los acontecimientos del mundo se derivan por causalidad natural, esto es, por causas sujetas a las leyes naturales, negando por tanto toda causalidad por libertad y toda posible causa incondicionada. Esto supone una serie infinita de causas.

Acudiendo a la noción de enlace dinámico, Kant evita rechazar ambas posiciones. La solución que ofrece Kant consiste en reconocer que la síntesis categorial pensada en esta antinomia -causalidad-permite llevar a cabo un enlace dinámico y no sólo homogéneo (como en el caso de la primera y segunda antinomia). A diferencia de lo que ocurre en estas dos, en la tercera antinomia podemos pensar el enlace causal de elementos no homogéneos. Así, mientras el realista trascendental confunde fenómeno y noúmeno y, por tanto, no puede sino pensar el enlace causal entre un único tipo de entidad ${ }^{13}$, Kant puede buscar otra solución. Puede admitir un tipo de causa del mundo que sea incondicionada y otro tipo de causa que no lo sea. En efecto, para conformar las pretensiones de tesis y antítesis, Kant propone una causa incondicionada fuera del tiempo, es decir heterogénea respecto del mundo (fenoménico), y una legalidad natural insoslayable (causalidad natural) referida al enlace entre fenómenos existentes únicamente en el tiempo.

Ahora bien, admitir semejante solución supone reformular el problema planteado. Si Kant tuviera que responder al pleito entre tesis y antítesis en los exactos

\footnotetext{
${ }^{11}$ A444/B472.

${ }^{12}$ A445/B473.

${ }^{13}$ Resulta especialmente llamativo que Grier no haya advertido esto. Ella supone que la tesis postula una causa libre fuera del tiempo. Si el realista trascendental no distingue adecuadamente fenómeno existente únicamente bajo la forma del tiempo- y cosa en sí -presuntamente existente con independencia de las formas de la sensibilidad-, difícilmente podría éste representarse la posibilidad de una causa fuera del tiempo (esto es, distinta en su género de los fenómenos). La autora interpreta de esta manera la tesis de la "Tercera antinomia", pues es uno de los elementos claves que ella utiliza para diferenciarla del "Cuarto conflicto cosmológico". Cfr. Op. Cit. GRIER, Michelle, Kant's doctrine of transcendental illusion, 220-221.
} 
términos en que este pleito es planteado, tendría dos posibilidades. Una de ellas es ser un realista trascendental más y oscilar entre la tesis y antítesis, sin poder hallar una solución al enigma. O bien podría descubrir el error del realismo trascendental y rechazar ambas afirmaciones por falsas (como parece sugerir en A505/B533) ${ }^{14}$. EI realismo trascendental supone que el mundo es una cosa en sí temporal y aquí se pregunta si tiene su origen en una primera causa temporal incondicionada o si consta de una serie infinita completa de causas temporales condicionadas. En tanto el mundo no es en sí, se daría por falsas ambas posiciones. Al introducir la distinción entre fenómeno y noúmeno, Kant no sólo resuelve la supuesta contradicción, sino que además reformula el problema.

Como vemos, el problema cosmológico de la tercera antinomia -en su formulación original- pierde todo sentido para el idealista trascendental: La idea cosmológica presupone como objeto una totalidad absoluta dada de condiciones del fenómeno y respecto a tal objeto se pregunta si tal serie absoluta contaba con una primera causa incondicionada (en virtud de la cual se afirmaba cierta causalidad por libertad) o si consistía en una serie infinita sujeta únicamente a la legalidad natural. Como esta serie no existe en sí misma, sino que sólo puede ser el producto de una síntesis empírica que se realiza sobre fenómenos dados a la intuición, responder la pregunta por una u otra posición se vuelve incoherente para el idealismo trascendental.

Al comprenderse que una serie de fenómenos se conforma únicamente por un regressus empírico (lo cual, sólo puede ocurrir al comprender la distinción entre fenómeno y noúmeno) puede entenderse que tal serie no es algo dado, sino que consta fundamentalmente de una actividad que se está ejecutando ${ }^{15}$. Respecto de tal serie, no tiene sentido preguntar si consta de un primer miembro incondicionado o si

\footnotetext{
14 "Lo que se ha dicho aquí de la primera idea cosmológica, a saber, de la totalidad absoluta de la cantidad en el fenómeno, vale también para todas las restantes. La serie de las condiciones sólo se encuentra en la síntesis regresiva misma, y no en sí, en el fenómeno, como en una cosa particular dada antes de todo regressus. Por eso, deberé también decir: la multitud de partes en un fenómeno dado no es, en sí, ni finita ni infinita, porque el fenómeno no es nada que exista en sí mismo, y las partes sólo son dadas mediante el regressus de la síntesis que descompone, y en él; regressus que nunca es dado de manera absolutamente íntegra ni como finito, ni como infinito. Eso mismo vale para la serie de las causas ordenadas unas sobre las otras [...]" A505/B533. La primera y la última cursiva son nuestras.

${ }^{15}$ A500-1/B528-9
} 
consiste en infinitas condiciones. Pues, la serie no está dada en sí misma, no está determinada por completo, sino que consiste de una actividad que se ejecuta sobre los fenómenos y es tan larga como cuán lejos se lleve a cabo el regressus. Este regreso que recorre fenómenos sucesivos no encuentra elemento alguno incondicionado en el que detenerse, pudiendo siempre proseguir la síntesis. Por este motivo, podría rechazarse la pretensión de la tesis. Pero, en oposición a la pretensión de la antítesis, este regressus no ofrece nunca una serie infinita completa. Afirmar, como lo hace la antítesis, la exclusividad de la causalidad natural para la totalidad de acontecimientos del mundo es un exceso, pues toma por supuesto que la serie es infinita y está completa, íntegramente dada.

Sin embargo, Kant no concluye que ambas pretensiones deban rechazarse, sino que reformulando el problema halla una solución. A este respecto, resultan especialmente interesantes las "Observaciones" a tesis y antítesis, pues ambas introducen elementos polémicos pero relevantes para la solución kantiana. La "Observación" a la tesis afirma que suponer una causalidad por libertad que diera origen al mundo implicaría reconocer la facultad de iniciar series causales durante el curso del mundo ${ }^{16}$. Esta afirmación ha hecho pensar a los intérpretes mencionados que el problema cosmológico sería abandonado en beneficio de un problema puramente práctico. La "Observación" a la antítesis recoge esta afirmación y la niega; pero agrega que en caso de admitirse una causalidad por libertad, sólo podría concederse una causa libre si ésta estuviera fuera del mundo (rechazándose por tanto toda facultad de iniciar series causales durante el curso del mundo) ${ }^{17}$. La solución kantiana se da en el entrecruzamiento de estos dos factores: se afirmará la posible compatibilidad entre la causalidad natural que sujeta irremediablemente a todos los fenómenos y la causalidad por libertad como causa nouménica -fuera del tiempo y por tanto indeterminada- de los fenómenos en el mundo (o de algunos fenómenos en particular).

Lo que nos interesa indicar aquí es que esta reformulación del problema implica que se continúa preguntando por la causa que origina el mundo, pero ahora referida a

\footnotetext{
${ }^{16} \mathrm{~A} 450 / \mathrm{B} 478$

${ }^{17}$ A451/B479
} 
los fenómenos en singular. Ya no se pregunta por la causa de la totalidad que conformaría al mundo -pues, tal totalidad no existe-, sino por la de cada fenómeno singular. La respuesta kantiana afirmará que podría admitirse la sujeción de todo fenómeno singular a la causalidad por naturaleza y, a la vez, suponer una causa nouménica suya. Esto implica la admisión del inicio de una serie durante el curso del mundo en lo que respecta a su causa nouménica, mientras el mismo fenómeno, en lo que respecta a la sucesión temporal, se encuentra sujeto a la necesidad natural y condicionado por los eventos precedentes.

La llamada "reformulación" del problema cosmológico consiste, entonces, en convertir el problema de la causa del mundo como una totalidad dada en la pregunta por la causa nouménica de los fenómenos tomados en particular. Mientras en la tercera antinomia los rivales debatían la existencia o inexistencia de una causa incondicionada temporal del mundo como un todo, la solución kantiana comienza por desarticular el problema y refomularlo. La pregunta por la causa (incondicionada o condicionada, libre o natural) pasa a ser respecto al fenómeno concreto y singular y no del mundo como totalidad.

El problema abordado en la solución a la tercera antinomia es éste reformulado. En efecto, ya no se vuelve a mencionar al mundo ni a preguntar por su origen. Se pregunta por una causa incondicionada y libre, origen de ciertos efectos o acontecimientos en el mundo ${ }^{18}$. Esto ha hecho pensar a los intérpretes mencionados que el problema cosmológico ha sido abandonado. Pero, como hemos mostrado, este abordaje consiste en una reformulación de un problema que no ha perdido su sentido cosmológico, pues se sigue preguntando por la causa u origen del mundo, aunque ya no como una totalidad dada, sino con respecto a cada fenómeno que constituye al mundo.

\footnotetext{
18 “[E]n la cuestión acerca de naturaleza y libertad encontramos ya la dificultad de si acaso la libertad será, en geenral, posible, y en caso de que lo sea, si puede ser compatible con la universalidad de la ley natural de la causalidad; y por tanto, [encontramos la dificultad de establecer] si será una proposición verdaderamente disyuntiva la [que dice] que todo efecto en el mundo debe surgir o bien por naturaleza, o bien por libertad, o si no podrán tener lugar a la vez ambas cosas en diferente respecto, en uno y el mismo acontecimiento. [...] [S] e plantea la pregunta de si, sin embargo, con respecto al mismo efecto que está determinado según la naturaleza, pudiera acaso tener lugar también la libertad" A536/B564. Las cursivas en "todo efecto en el mundo", "en uno y el mismo acontecimiento" y en "mismo efecto" son nuestras, las restantes son del autor.
} 


\section{Sobre la razón en la solución kantiana al problema cosmológico}

La solución de la tercera antinomia se divide en tres secciones. La primera sección consiste fundamentalmente en la reformulación del problema en cuestión. Sin embargo, para probar que el tratamiento de la tercera antinomia tiene un carácter estrictamente cosmológico no resulta suficiente esta reformulación. Es necesario mostrar que la temática que se desarrolla en toda la solución de la antinomia es cosmológica. La mayor objeción que puede hacerse a esta posición podría consistir en destacar los términos con los que Kant se expresa aquí. Mientras la segunda parte de esta solución consiste esencialmente en la presentación de los requisitos generales que deben ser satisfechos para que sea posible la compatibilidad entre la causalidad por naturaleza y la causalidad por libertad, la tercera parte prueba que tales requisitos podrían, en efecto, ser satisfechos. Aquí entran en juego los conceptos de "razón práctica" y "libertad práctica”. La introducción de la razón práctica, la libertad práctica y el libre albedrío es, por un lado, un elemento clave en la solución del problema cosmológico. Aunque también es, por el otro lado, el motivo fundamental por el cual se ha creído erróneamente que Kant se ocupa de un problema puramente práctico.

En lo que resta de nuestro trabajo debe explicarse por qué Kant introduce el tratamiento de la razón práctica y la libertad práctica para resolver el problema cosmológico, y qué función cumplen estos elementos en la argumentación. Esto no implica bajo ningún punto de vista rechazar que la prueba de la posible compatibilidad de ambas causalidades tenga una relevancia práctica. Ella abre la posibilidad del abordaje propiamente práctico de la libertad, sin que tal tratamiento implique una ruptura del orden natural. No obstante, consideramos que la solución estudiada tiene un lado estrictamente cosmológico.

Comencemos por la segunda parte de la solución. En esta segunda parte se presentan los requisitos generales para la resolución del problema. Para ello resulta fundamental la distinción entre carácter empírico e inteligible. Un sujeto, además de su relación causal con los fenómenos de acuerdo con la ley de su causalidad fenoménica, i.e. su carácter empírico, puede tener una facultad no sensible, i.e. 
inteligible. Esta facultad podría tener una aptitud causal respecto de los fenómenos sin ser ella misma fenoménica. Tal aptitud causal implicaría un carácter inteligible, una legalidad de su causalidad inteligible. En virtud de ser una facultad inteligible que actúa causalmente sobre los fenómenos sin estar ella misma condicionada por la sucesión temporal, tal facultad sería incondicionada y libre en su causalidad. De cumplirse todos estos requisitos, los fenómenos que fueran efecto de esta facultad se encontrarían sujetos a dos tipos diferentes de causalidad: la causalidad según la legalidad de la naturaleza en tanto existen en el tiempo y son precedidos por otros eventos y la causalidad de aquella causa inteligible, incondicionada y libre. Si resultara posible que cierto fenómeno se encuentre sujeto a ambas causalidades, entonces se probaría la posibilidad de la compatibilidad entre naturaleza y libertad. Como puede verse, esta descripción general de una solución posible concuerda con el problema "reformulado" de la tercera antinomia. Es decir, no se pregunta por la causa del mundo como una totalidad, sino de los fenómenos singulares (de cada uno) que lo componen. Analizar la causa (libre o natural) de cada fenómeno particular permite advertir si su origen es incondicionado o no.

La tercera parte de la solución debe mostrar la posiblidad de satisfacer estos requisitos. Debe mostrarse la posibilidad de que un ser tenga (1) una facultad inteligible (2) con aptitud causal respecto de los fenómenos y (3) cuyo carácter inteligible se encuentre en relación con su carácter empírico. Precisamente, el hombre será aquella entidad que se presentará como satisfaciendo estos requisitos. El hombre, que a través de la apercepción empírica se piensa como más allá de lo sensible y descubre en sí facultades inteligibles (razón y entendimiento), es la entidad que podría satisfacer estos requisitos ${ }^{19}$.

19 "En la naturaleza inanimada, o que tiene vida meramente animal, no encontramos ningún fundamento para concebir alguna facultad que no sea condicionada de manera meramente sensible. Sólo el hombre, que por otra parte conoce a toda la naturaleza únicamente por los sentidos, se conoce a sí mismo también por mera apercepción; y [se conoce así] en acciones y en determinaciones internas que no puede contar entre las impresiones de los sentidos, y es para sí mismo, por un lado, ciertamente, fenómeno, pero por otro lado, a saber, con respecto a ciertas facultades, [es] un objeto meramente inteligible, porque la acción de él no puede contarse entre lo que pertenece a la receptividad de la sensibilidad. A esas facultades las llamamos entendimiento y razón" A546/B574. 
Ahora bien, frente al incuestionado carácter empírico del hombre como un ser fenómenico y natural, debe alegarse a favor de la naturaleza inteligible de la razón y su aptitud causal. Esto es, debe explicarse (1) el carácter inteligible del hombre, (2) la relación de sus caracteres inteligible y empírico y (3) la aptitud causal del hombre sobre los fenómenos en tanto ser inteligible.

Respecto de la primera cuestión mencionada, Kant indica que "la razón, en su causalidad, no está sometida a ninguna de las condiciones del fenómeno ni del curso del tiempo". También sostiene que la razón "no está en el tiempo, ni cae en un estado nuevo en el que antes no estaba" ${ }^{20}$. En el mismo sentido, Kant afirma que

[e]n lo que se refiere al carácter inteligible [...] no tiene validez ningún antes, ni ningún después y toda acción, independientemente de la relación de tiempo en la que está junto con otros fenómenos, es el efecto inmediato del carácter inteligible de la razón pura, la cual, por tanto, actúa libremente, sin estar determinada dinámicamente en la cadena de las causas naturales por fundamentos externos ni internos que precedan según el tiempo (A553/B581).

De acuerdo con esto, la razón es presentada como aquella facultad inteligible fuera del tiempo y exenta a la determinación causal propia de los fenómenos. Esta facultad incondicionada es el fundamento del libre albedrío mencionado en la primera parte de la solución. La acción libre del hombre, basada en la facultad inteligible, es el modo kantiano de responder a la pregunta por el origen incondicionado de ciertos fenómenos.

Teniendo en cuenta que Kant ha caracterizado a la razón como una facultad inteligible que podría ser causa incondicionada de ciertos fenómenos, resulta necesario considerar tal aptitud causal. Para ello es necesario analizar los conceptos de libre albedrío y libertad. Kant define al albedrío del hombre como "liberum, porque la sensibilidad no hace necesaria la acción de él, sino que en el ser humano reside una facultad de determinarse por sí mismo, independientemente de la coacción ejercida por móviles sensibles"21. En concordancia con esta definición, Kant caracteriza la

\footnotetext{
${ }^{20}$ A556/B584

${ }^{21}$ A534/B562
} 
libertad en sentido práctico como "la independencia del albedrío, respecto de la coacción por impulsos de la sensibilidad" ${ }^{22}$, diferenciándola de la libertad "en sentido cosmológico" entendiendo por ella "la facultad de comenzar por sí mismo un estado, la causalidad de la cual no está a su vez, según la ley de la naturaleza, sometida a otra causa que la determine según el tiempo"23. A esta última, cuya definición concuerda con la idea de libertad trascendental en A446/B474, Kant la llama "idea trascendental pura" e indica que sobre ésta "se funda el concepto práctico" de la libertad ${ }^{24}$. Unas líneas más abajo Kant indica que "la supresión de la libertad trascendental aniquilaría a la vez toda libertad práctica" ${ }^{25}$.

El problema tal como originalmente fue planteado en la tercera antinomia se refiere a la libertad trascendental, a aquella caracterizada por Kant como libertad "en sentido cosmológico", esto es, la pregunta por la facultad de comenzar por sí mismo un estado. Así, la pregunta por el origen del mundo sería respondida admitiendo tal libertad ( $y$, en consecuencia, admitiendo una causa libre o incondicionada en la cadena de causas que conforma el mundo) o rechazándola (admitiendo, entonces, que el mundo es una serie de causas infinita). La introducción de la libertad práctica, presentada como el no condicionamiento sensible del albedrío humano, parece sacarnos del ámbito cosmológico. Sin embargo, es coherente con la reformulación kantiana del problema cosmológico.

Como hemos indicado, el problema cosmológico "reformulado" pregunta por la causa incondicionada de cada fenómeno. La libertad práctica permite resolver el problema cosmológico, pues permite pasar de la pregunta metafísico-dogmática por la causa incondicionada del mundo como un todo al tratamiento idealista trascendental de la causa incondicionada de un fenómeno en particular, la acción humana. En otras palabras, el abordaje de la libertad práctica es el modo que encuentra Kant para

\footnotetext{
${ }^{22}$ A534/B562

${ }^{23}$ A533/B561. De igual modo, en A449/B477 Kant se refiere a "aquello que ha puesto siempre en tanta perplejidad a la razón especulativa, es propiamente [algo] sólo trascendental, y se refiere solamente a si se debe admitir una facultad de comenzar por sí una serie de cosas o de estados sucesivos". En A446/B474 se caracteriza a la libertad trascendental como "una espontaneidad absoluta de las causas, capaz de comenzar por sí misma una serie de fenómenos".

${ }^{24}$ A533/B561

${ }^{25}$ A534/B562
} 
responder la pregunta por la causa incondicionada, ya no del mundo como una totalidad absoluta dada, sino de cada fenómeno que compone al mundo. Así, en vez de preguntar por la causa del mundo como un todo y buscar una causa incondicionada al comienzo de la serie de fenómenos, se pregunta por la causa incondicionada atemporal de cada fenómeno en particular (o, al menos, de cierto tipo de fenómeno: la conducta humana).

Junto al abordaje del libre albedrío, el texto introduce una mención de la razón pura en su uso práctico. Resulta innegable que la libertad está en íntima relación con la razón en su uso práctico, toda vez que ésta es la facultad de determinar la facultad de desear con independencia del condicionamiento sensible. No obstante, Kant introduce esta referencia a la razón práctica por un motivo concreto: El filósofo no pretende estudiar aquí la razón práctica, sino sólo alegar a favor de la existencia de una aptitud causal de la razón, para lo cual ofrece buenas razones basadas en la conciencia del deber.

Veamos esto al tratarse sobre la relación entre el carácter empírico y el carácter inteligible ${ }^{26}$. Mientras el carácter empírico se presenta en el hombre como su manera de sentir, el carácter inteligible es caracterizado como su manera de pensar, la cual coincide con la descripción de aquella facultad ajena al tiempo e incondicionada. Kant describe la relación entre la manera de pensar (carácter inteligible del hombre) y la manera de sentir (carácter empírico) del siguiente modo:

Éste [el carácter empírico] está determinado a su vez en el carácter inteligible (en la manera de pensar). Pero esta última no la conocemos, sino que la designamos por medio de fenómenos, que propiamente sólo dan a conocer inmediatamente el modo de sentir (el carácter empírico). Ahora bien, la acción, en la medida en que ha de atribuirse a la manera de pensar, como [si la manera de pensar fuera la] casua de ella, no resulta de ella según leyes empíricas, es decir, de tal manera, que las condiciones de la

\footnotetext{
${ }^{26}$ A539/B567 "[T] oda causa eficiente debe tener un carácter, [...], una ley de su causalidad, sin la cual ella no sería causa. Y entonces, tendríamos en un sujeto del mundo sensible, primeramente, un carácter empírico, por el cual sus acciones, como fenómenos, estarían en íntegra interconexión con otros fenómenos, según leyes naturales constantes [...]. En segundo lugar habría que otorgarle [a ese sujeto] también un carácter inteligible por el cual él es, ciertamente, la causa de aquellas acciones [...]". El carácter es la legalidad de la causalidad de la que se trate en cuestión, la aptitud causal misma de la causa.
} 
razón pura precedan, sino sólo de tal manera, que los efectos de ella preceden en el fenómeno del sentido interno (A551/B579).

A partir de esta descripción compleja, entendemos que (1) el carácter inteligible (fuera del tiempo) causa el carácter empírico (que rige la causalidad fenoménica de este ente); (2) siendo el carácter inteligible la manera de pensar y el carácter empírico la manera de sentir, la manera de pensar (incondicionada por la sensiblidad) causa y determina la manera de sentir; (3) la manera de pensar no precede a la acción, pero esta acción sí es precedida por los efectos que la manera de pensar causa en la manera de sentir (fenómeno en el sentido interno).

Kant parece identificar "manera de sentir" o "carácter empírico" del hombre con "sentido interno" o al menos con ciertos "fenómeno[s] del sentido interno". Entiendo que tales fenómenos concordarían con lo que habitualmente llamamos "motivos" de la acción. La manera de sentir o fenómeno en el sentido interno tendría una causa nouménica en la manera de pensar. Este efecto de la manera de pensar tiene una relación causal con la acción, en concordancia con las leyes naturales. Es por esto que Kant afirma que "si pudiéramos examinar hasta el fondo todos los fenómenos del albedrío de él [el hombre], no habría ni una sola acción humana que no pudiéramos predecir con certeza y que no pudiéramos conocer como necesaria a partir de sus condiciones previas" ${ }^{27}$. Estos fenómenos del albedrío a los que se refiere Kant son los antes llamados efectos de la razón pura en el sentido interno. Si, en efecto, como Kant indica, conociéramos estos fenómenos del sentido interno (motivos) y las restantes causas concomitantes, podríamos predecir la acción sin dejar de atribuirle libertad, en tanto esta acción en relación con la razón es incondicionada ${ }^{28}$ y en la medida en que esta razón es la causa del carácter empírico ${ }^{29}$.

Como vemos, esta explicación concuerda con el esquema general de la solución presentado en la segunda parte. Pero, esta vez, aplicado a la única entidad a que se le

\footnotetext{
${ }^{27}$ A550/B578.

28 “Pero si consideramos esas mismas acciones en relación con la razón [...] éstas no han acontecido porque estuvieran determinadas por causas empíricas, no, sino porque estaba determinadas por fundamentos de la razón" A550/B578.

29 “Éste [el carácter empírico] está determinado a su vez en el carácter inteligible (en la manera de pensar)." A551/B579.
} 
atribuye una facultad inteligible y una causalidad fenoménica: el hombre. También concuerda con aquella afirmación de Kant en que se presenta al carácter empírico siendo determinado por el carácter inteligible ${ }^{30}$.

Volvamos a considerar la aptitud causal de la razón. Como es sabido, Kant menciona reiteradas veces a la razón práctica y el deber en la tercera parte de la solución de esta antinomia. Estas menciones son en parte responsables de haber hecho creer que Kant abordaría aquí una temática eminentemente práctica. Sostenemos, no obstante, que la mención de la razón práctica en este contexto se explica por un motivo estrictamente argumental.

Kant introduce aquí a la razón práctica únicamente para probar que la razón podría tener la aptitud causal que se debe suponer para admitir un carácter inteligible. Kant no está interesado -al menos, no aquí- en demostrar que la razón pura en su uso práctico es el asiento de la libertad práctica. Esto sí sería tema propio de la filosofía práctica. Kant pretende mucho menos: debe demostrar que el tercer requisito para admitir la compatibilidad de ambas causalidades es admisible. Ni siquiera se necesita demostrar que la libertad es un hecho efectivo. Lo único que se necesita demostrar en este contexto es que sería admisible la causalidad de la razón sobre los fenómenos. Para ello, sólo se necesita alegar que, generalmente, admitimos que la razón humana puede determinar nuestra conducta. Y el mejor ejemplo para alegar esto es referir a la determinación de la conducta a través de la conciencia del deber.

Evidentemente, esta determinación de la conducta por parte de la razón debe hacer referencia a la razón en su uso práctico. Sin embargo, no es el tema aquí abordado. De hecho, Kant no se ocupa aquí de probar que sólo la razón pura práctica (determinada por principios morales) es aquella facultad donde radica la libertad. Allison afirma que aquí se trata de forma más amplia a la razón, incluyendo todo acto espontáneo suyo que pueda autodeterminarla ${ }^{31}$. Así, la "manera de pensar" -que causa al carácter empírico y a los "fenómeno[s] del sentido interno" que provocan la acción- podría no ser necesariamente la razón práctica en sentido estricto (la razón

30 "[...] sólo por medio del carácter empírico (que es el mero fenómeno del inteligible)" A541/B569. La cursiva es nuestra.

${ }^{31}$ Op. Cit. ALLISON, Henry, El idealismo trascendental de Kant: una interpretación y defensa, 488 y Op. Cit. Allison, Henry, Kant's theory of freedom, 37-39. 
pura práctica). Insistimos, Kant no necesita probar que la razón práctica es el fundamento de la libertad. Sólo necesita demostrar que hay buenos motivos para creer que la razón puede determinar la conducta. Y para lograr esto, se refiere a la conciencia del deber: la conciencia del deber nos da un ejemplo concreto en el que, habitualmente, admitimos que la razón guía a la conducta.

Así, tales menciones de la razón práctica son introducidas como alegatos a favor de la aptitud causal de la razón sobre los fenómenos. Veamos brevemente estas referencias. Kant sostiene

\begin{abstract}
que esta razón tiene causalidad, o al menos, que nos representamos una [causalidad] en ella, es algo que está claro en virtud de los imperativos que, como reglas, les imponemos, en todo lo que es práctico, a las potencias que [en ello] operan. El [verbo en infinitivo] deber expresa una especie de necesidad y de conexión con fundamentos, que no se presenta en toda la naturaleza. [...] Este "deber" expresa una acción posible, cuyo fundamento no es nada más que un mero concepto (A547/B575).
\end{abstract}

Como puede verse, este párrafo comienza afirmando "que esta razón tiene causalidad"; de igual modo, el párrafo siguiente termina afirmando que la razón

\begin{abstract}
se fabrica, con entera espontaneidad, un orden propio según ideas, en las que acomoda las condiciones empíricas, y según las cuales ella incluso declara necesarias unas acciones que sin embargo no han ocurrido y que quizá no ocurran nunca, pero de las cuales presupone empero que la razón pudiera tener causalidad con respecto a ellas; pues sin eso no podría esperar, de sus ideas, efectos en la experiencia (A548/B576) ${ }^{32}$.
\end{abstract}

Luego de esto, Kant afirma "detengámonos ahora aquí, y admitamos, al menos como posible, que la razón tiene efectivamente causalidad con respecto a los fenómenos". Vemos con claridad que los párrafos antes mencionados fueron introducidos con este propósito: ofrecer buenas razones para creer que la razón tiene cierta causalidad sobre los fenómenos y no para explicar bajo qué principios determina la razón pura a la voluntad. Veamos otra mención relevante de Kant de la razón práctica.

\footnotetext{
${ }^{32}$ La cursiva en "presupone" es nuestra.
} 
[S]i consideramos esas mismas acciones en relación con la razón, y ciertamente, no [en relación con] la razón especulativa, para explicarlas en lo que respecta al origen de ellas, sino [cuando las consideramos] solamente en la medida en que la razón es la causa de la generación de ellas; en una palabra, si las confrontamos con ésta atendiendo a lo práctico, encontramos una regla y un orden muy diferentes del orden de la naturaleza (A550/B578).

Luego de esta afirmación Kant comienza el próximo párrafo diciendo "supuesto que se pudiera decir: la razón tiene causalidad con respecto al fenómeno". Nuevamente, según estas palabras, debemos creer que el párrafo antes citado ha sido introducido por Kant para alegar a favor de la causalidad de la razón sobre los fenómenos y no para explicar la determinación racional (pura práctica) de la conducta.

Como vemos, estas menciones no intentan probar que el carácter inteligible que causa libremente la acción es la razón pura en su uso práctico stricto sensu. Estas referencias al deber y a la razón práctica intentan ofrecer buenos motivos para reconocer que la razón o "manera de pensar" tiene una aptitud causal sobre el fenómeno, sin que sea necesario establecer la naturaleza de los principios por medio de los cuales la razón puede determinar a priori a la voluntad.

Así, aquí Kant no prueba -ni sería necesario que lo haga aquí- que la razón pura es capaz de determinar a la facultad de desear por medio de principios que deben ser imperativos categóricos o leyes prácticas ${ }^{33}$. No es necesario demostrar que esta manera de pensar -causa de la acción- es determinada necesariamente por la conciencia del deber moral. En este sentido, sería admisible la sugerencia de Allison que, como vimos, indica que Kant está pensando de manera más amplia la capacidad de la razón para determinarse por principios inteligibles. Esta determinación incluiría cualquier acto de espontaneidad de la razón según el cual ésta se determine por

\footnotetext{
${ }^{33}$ Granja Castro es muy clara al indicar que el fin de la Crítica de la razón práctica es "establecer la existencia de una razón pura práctica y, a partir de ella, la existencia de la libertad trascendental", esto es, demostrar que "la razón es práctica en sí misma, i.e., que es capaz de proporcionar los motivos y fines para obrar" (GRANJA CASTRO DE PROBERT, Dulce María, "Introducción", Kant, Immanuel, Crítica de la razón práctica, Buenos Aires, Colihue, 2013, pp XIX y XXI). Como vemos, la investigación de la razón pura práctica no es objeto de la tercera antinomia, sino de la Crítica de la razón práctica.
} 
principios inteligibles ${ }^{34}$. La razón, al autodeterminarse con total independencia de la sensibilidad, sería una causa incondicionada. En lo que hace a nuestros intereses, no es necesario decidir si esta facultad se caracteriza por una espontaneidad en su autodeterminación teórica o si se limita a la razón pura en su uso práctico. Nos alcanza con comprender que Kant introduce la determinación racional de la conducta para dar por probada la aptitud causal de esta facultad.

\section{Conclusiones}

La solución de la tercera antinomia se compone de tres secciones. La primera reformula el problema cosmológico metafísico-dogmático en términos acordes con el idealismo trascendental. Esta reformulación hacía que el problema en cuestión siga siendo cosmológico, aunque ya no referido al origen del mundo como una totalidad, sino a la causa u origen de cada fenómeno en particular (o algunos de ellos). La segunda parte presenta los requisitos generales exigibles para la admisión de la posibilidad de una causalidad incondicionada compatible con la causalidad por naturaleza. La tercera parte muestra que dichos requisitos pueden ser efectivamente satisfechos por un ente, el hombre.

En el marco general de esta interpretación según la cual el problema y su solución conservan en todo caso un sentido cosmológico, debía explicarse por qué Kant introducía referencias explícitas sobre la libertad y la razón prácticas. El abordaje de Kant de la libertad práctica en la primera parte es el modo a través del cual se reformula y se resuelve el problema originariamente abordado en términos de libertad trascendental. La introducción de la libertad práctica es la pieza clave para la resolución del problema cosmológico en los legítimos téminos del idealismo trascendental. Junto a este estudio de la libertad práctica se introduce una consideración sobre la razón. Esta razón es caracterizada como una facultad inteligible, con aptitud causal nouménica sobre el fenómeno y en relación y compatibilidad con el

\footnotetext{
34 “[L]a razón no cede a aquel fundamento que es empíricamente dado, ni sigue el orden de las cosas tal como éstas se presentan en el fenómeno, sino que se fabrica, con entera espontaneidad, un orden propio según ideas, en las que acomoda las condiciones empíricas" A548/B576. La cursiva es nuestra.
} 
carácter empírico del hombre. La razón, como aquella facultad capaz de determinarse con independencia de los condicionantes sensibles, se identifica con el libre albedrío o la libertad práctica a la que se refiere el texto y es la causa incondicionada de ciertos fenómenos. El estudio estricto de esta facultad exigiría caracterizarla como razón pura en su uso práctico. Sin embargo, hemos mostrado que éste no es el lugar en el que Kant se esté ocupando de determinar la naturaleza precisa de la razón que puede operar como causa incondicionada de los fenómenos. Las referencias a la razón práctica se explicaban por una necesidad argumental de Kant de dar por probada la aptitud causal de la razón sobre los fenómenos. Probada tal aptitud causal, ciertos fenómenos (particularmente, la conducta humana) tendrán una causa inteligible compatible con la causalidad fenoménica que los sujeta según la sucesión temporal. Con la introducción del libre albedrío y analizando la facultad inteligible detrás suyo, se resuelve la pregunta cosmológica por la causa incondicionada, aunque referida al origen incondicionado de ciertos fenómenos concretos en particular. Y con ello se resuelve también la posible compatibilidad de ésta con la causalidad por naturaleza. 